PROCEEDINGS OF THE

AMERICAN MATHEMATICAL SOCIETY

Volume 139, Number 5, May 2011, Pages 1707-1716

S 0002-9939(2010)10602-0

Article electronically published on October 29, 2010

\title{
ON A QUESTION OF J. BORWEIN AND H. WIERSMA
}

\author{
BOIL MUSEV AND NADEZHDA RIBARSKA
}

(Communicated by Nigel J. Kalton)

\begin{abstract}
We present an explicit example of an acyclic operator with bounded domain, thus answering a question of Borwein and Wiersma.
\end{abstract}

\section{INTRODUCTION}

A valuable tool in the study of various mappings of importance in analysis is the following concept of monotonicity.

Definition 1. Let $X$ be a Banach space and $X^{*}$ be its topological dual. A multivalued mapping $T: X \Rightarrow X^{*}$ is a monotone operator if

$$
\left\langle x^{*}-y^{*}, x-y\right\rangle \geq 0
$$

whenever $x^{*} \in T(x)$ and $y^{*} \in T(y)$. The monotone operator $T$ is said to be maximal monotone if $g r T \subset g r S$ with $S$ monotone implies $T=S$. The set

$$
\operatorname{dom}(T):=\{x \in X: T(x) \neq \emptyset\}
$$

is said to be the domain of the monotone operator $T$.

The most popular, broadly studied and well behaved subclass of the class of all maximal monotone operators is the class of subdifferential mappings. Let us recall that if $f$ is a proper convex lower semicontinuous function on $X$, then its subdifferential is the mapping

$$
\partial f(x):=\left\{x^{*} \in X:\left\langle x^{*}, y-x\right\rangle+f(x) \leq f(y) \text { for all } y \in X\right\} .
$$

A classical theorem of Rockafellar (see 4, 5) states that subdifferentials of convex functions are exactly the maximal cyclically monotone operators. There are many problems that have been solved for such mappings and still remain open for arbitrary monotone operators. We would like to mention just two of them. Is it true that monotone operators are generically single-valued on a weak Asplund space (that is, where subdifferential mappings are generically single-valued)? Is it true that a differential inclusion on an open subset of $R^{n}$ with an upper semicontinuous

Received by the editors September 24, 2009 and, in revised form, May 12, 2010 and May 20, 2010

2010 Mathematics Subject Classification. Primary 47H04, 52A41.

Key words and phrases. Monotone operators, cyclic monotonicity, acyclic operators.

The first author was partially supported by the Bulgarian National Fund for Scientific Research under contract DO 02-360/2008.

The second author was partially supported by the Bulgarian National Fund for Scientific Research under contract DO 02-360/2008.

(C)2010 American Mathematical Society 
autonomous monotone right-hand side has a local solution? (The respective result is true for cyclic monotone upper semicontinuous operators.) Thus it is natural to consider decompositions of the form $T=\partial f+R$ with the idea that $\partial f$ is the "nice" part of $T$ and the remainder $R$ is the "trouble-maker".

Definition 2. Given a set $C \subset R^{n}$, a monotone single-valued operator $T: R^{n} \rightarrow$ $R^{n}$ is said to be acyclic with respect to $C$ if $T=\partial f+R$ with $R$ monotone implies that $\partial f$ is constant on $C$. If no set $C$ is specified, then $C=\operatorname{dom} T$ is implied.

One may think that acyclic operators are those monotone mappings which do not contain a nontrivial cyclic part. A modernized version of a 1970 result of Asplund (see [1, 2], 3]) shows that every monotone operator can be decomposed into a subdifferential mapping and an acyclic operator.

Theorem 1 (cf. [3). Suppose we are given a (single-valued) maximal monotone operator $T: \operatorname{dom} T \subset R^{n} \rightarrow R^{n}$ with int $\operatorname{dom} T \neq \emptyset$. Then $T$ may be decomposed as

$$
T=\nabla f+R,
$$

where $f$ is lower semicontinuous and convex, while $R$ is acyclic with respect to domT.

The first explicit example of a nonlinear acyclic operator appears in 3 . This example is shown to be acyclic using the concrete form of the mapping. No method is known to check whether or not an operator is acyclic. In the paper [3] the following question was asked:

Question. Can one exhibit an acyclic mapping whose domain is not the whole space?

In this paper we give a positive answer to this question. The example we present is

$$
S(x)=\left(\sqrt{-\frac{1}{6(\ln \|x\|)^{3}}-\frac{1}{2(\ln \|x\|)^{2}}-\frac{1}{2 \ln \|x\|}}\right) \cdot \frac{x}{\|x\|}-\frac{1}{\ln \|x\|} \cdot \frac{\left(-x_{2}, x_{1}\right)}{\|x\|}
$$

away from the origin and $S(0,0)=(0,0)$. In a sense this example has exactly opposite properties with respect to the example presented by Borwein and Wiersma. Its domain is the open unit disc, and its range is the whole plane; compare this with the Borwein-Wiersma example, whose domain is the whole plane and whose range is the unit disc.

Although far from being general, our approach provides a method for identifying the acyclic mappings in the (very small) class of radially symmetric monotone operators on the plane.

\section{Building AN EXAMPle}

We are going to seek examples of acyclic monotone operators in the class of radially symmetric operators on the plane.

Definition 3. We call an operator $S: \operatorname{dom} S \subset R^{2} \rightarrow R^{2}$ (the domain of $S$ could be a disc in $R^{2}$ or the whole plane) radially symmetric if it has the appearance

$$
S(x)=\varphi(\|x\|) \frac{x}{\|x\|}+\psi(\|x\|) \frac{\operatorname{rot}(x)}{\|x\|},
$$


where $\operatorname{rot}(x)=\operatorname{rot}\left(x_{1}, x_{2}\right):=\left(-x_{2}, x_{1}\right), \varphi$ and $\psi:(0, a) \longrightarrow R$ are smooth singlevalued functions $(a>0$ or $a=+\infty)$ and $\varphi$ is continuous at zero. We assume that $S(0,0)=(0,0)$ and $\varphi(0)=0$.

To ensure monotonicity of an operator of this class we use the following well known monotonicity test for differentiable mappings:

Proposition 1 (cf. 12.3 on p. 534 in [5]). A differentiable mapping $S: R^{n} \rightarrow R^{n}$ is monotone if and only if for each $x$ the Jacobian matrix $\nabla S(x)$ (not necessarily symmetric) is positive-semidefinite.

Let $x$ be any vector in the domain of $S$ not coinciding with the origin, and let us assign to it the orthogonal coordinate system

$$
\left(e_{1}(x), e_{2}(x)\right):=\left(\frac{x}{\rho}, \frac{\operatorname{rot}(x)}{\rho}\right),
$$

where $\rho:=\|x\|$. Then we calculate the Jacobian matrix of $S$ at the point $x$ with respect to the coordinates $\left(e_{1}(x), e_{2}(x)\right)$. First note that $\left\|x+t e_{1}(x)\right\|=\rho+t$,

$$
\frac{x+t e_{1}(x)}{\left\|x+t e_{1}(x)\right\|}=\frac{x}{\rho} \quad \text { and } \quad \frac{\operatorname{rot}\left(x+t e_{1}(x)\right)}{\left\|x+t e_{1}(x)\right\|}=\frac{\operatorname{rot}(x)}{\rho} .
$$

Then

$$
S\left(x+t e_{1}(x)\right)-S(x)=(\varphi(\rho+t)-\varphi(\rho)) \frac{x}{\rho}+(\psi(\rho+t)-\psi(\rho)) \frac{\operatorname{rot}(x)}{\rho}
$$

and, hence,

and

$$
\lim _{t \rightarrow 0} \frac{\left\langle S\left(x+t e_{1}(x)\right)-S(x), e_{1}(x)\right\rangle}{t}=\varphi^{\prime}(\rho)
$$

$$
\lim _{t \rightarrow 0} \frac{\left\langle S\left(x+t e_{1}(x)\right)-S(x), e_{2}(x)\right\rangle}{t}=\psi^{\prime}(\rho) .
$$

Now $\left\|x+t e_{2}(x)\right\|=\sqrt{\rho^{2}+t^{2}}$ yields

$$
\begin{aligned}
S & \left(x+t e_{2}(x)\right)-S(x) \\
= & \varphi\left(\sqrt{\rho^{2}+t^{2}}\right) \frac{x+t e_{2}(x)}{\sqrt{\rho^{2}+t^{2}}}+\psi\left(\sqrt{\rho^{2}+t^{2}}\right) \frac{\operatorname{rot}\left(x+t e_{2}(x)\right)}{\sqrt{\rho^{2}+t^{2}}}-\varphi(\rho) e_{1}(x)-\psi(\rho) e_{2}(x) \\
& =\left(\varphi\left(\sqrt{\rho^{2}+t^{2}}\right) \frac{\rho}{\sqrt{\rho^{2}+t^{2}}}-\psi\left(\sqrt{\rho^{2}+t^{2}}\right) \frac{t}{\sqrt{\rho^{2}+t^{2}}}-\varphi(\rho)\right) \frac{x}{\rho} \\
& +\left(\varphi\left(\sqrt{\rho^{2}+t^{2}}\right) \frac{t}{\sqrt{\rho^{2}+t^{2}}}+\psi\left(\sqrt{\rho^{2}+t^{2}}\right) \frac{\rho}{\sqrt{\rho^{2}+t^{2}}}-\psi(\rho)\right) \frac{\operatorname{rot}(x)}{\rho} .
\end{aligned}
$$

Therefore

$$
\begin{aligned}
& \lim _{t \rightarrow 0} \frac{\left\langle S\left(x+t e_{2}(x)\right)-S(x), e_{1}(x)\right\rangle}{t} \\
& \quad=\lim _{t \rightarrow 0} \frac{1}{t}\left(\varphi\left(\sqrt{\rho^{2}+t^{2}}\right) \frac{\rho}{\sqrt{\rho^{2}+t^{2}}}-\psi\left(\sqrt{\rho^{2}+t^{2}}\right) \frac{t}{\sqrt{\rho^{2}+t^{2}}}-\varphi(\rho)\right)=-\frac{1}{\rho} \psi(\rho), \\
& \lim _{t \rightarrow 0} \frac{\left\langle S\left(x+t e_{2}(x)\right)-S(x), e_{2}(x)\right\rangle}{t} \\
& \quad=\lim _{t \rightarrow 0} \frac{1}{t}\left(\varphi\left(\sqrt{\rho^{2}+t^{2}}\right) \frac{t}{\sqrt{\rho^{2}+t^{2}}}+\psi\left(\sqrt{\rho^{2}+t^{2}}\right) \frac{\rho}{\sqrt{\rho^{2}+t^{2}}}-\psi(\rho)\right)=-\frac{1}{\rho} \varphi(\rho) .
\end{aligned}
$$


Thus the Jacobian matrix of $S$ at the point $x$ with respect to the coordinates $\left(e_{1}(x), e_{2}(x)\right)$ is

$$
\nabla S(x)=\left(\begin{array}{rr}
\varphi^{\prime}(\rho) & -\frac{1}{\rho} \psi(\rho) \\
\psi^{\prime}(\rho) & \frac{1}{\rho} \varphi(\rho)
\end{array}\right) .
$$

The quadratic form generated by $\nabla S(x)$ is the same as the form generated by the symmetric matrix

$$
A(x)=\left(\begin{array}{cc}
\varphi^{\prime}(\rho) & \frac{1}{2}\left(\psi^{\prime}(\rho)-\frac{1}{\rho} \psi(\rho)\right) \\
\frac{1}{2}\left(\psi^{\prime}(\rho)-\frac{1}{\rho} \psi(\rho)\right) & \frac{1}{\rho} \varphi(\rho)
\end{array}\right) .
$$

Silvester's criterion yields that this matrix is positive-semidefinite if and only if the following two conditions hold true:

$$
\mid \begin{aligned}
& \varphi^{\prime}(\rho) \geq 0, \\
& 4 \rho \varphi^{\prime}(\rho) \varphi(\rho) \geq\left(\rho \psi^{\prime}(\rho)-\psi(\rho)\right)^{2} .
\end{aligned}
$$

Our guess is that a monotone radially symmetric operator could be acyclic if "we cannot subtract anything from the radial velocity", that is, if we actually have equality in the second of the above inequalities.

Definition 4. Let $S: \operatorname{dom} S \subset R^{2} \rightarrow R^{2}$ be a radially symmetric operator. We say that $S$ is a minimal monotone radially symmetric operator if

$$
\mid \begin{aligned}
& \varphi^{\prime}(\rho) \geq 0 \\
& 4 \rho \varphi^{\prime}(\rho) \varphi(\rho)=\left(\rho \psi^{\prime}(\rho)-\psi(\rho)\right)^{2}
\end{aligned}
$$

for every $\rho \in(0, a)$.

Note that the conditions imposed on $S$ in Definition 4 guarantee that $S$ is monotone. Indeed, $\varphi^{\prime}(\rho) \geq 0$ and $\varphi(0)=0$ imply that

$$
\langle S(x)-S(0,0), x-(0,0)\rangle=\rho \varphi(\rho) \geq 0,
$$

and Proposition 1 deals with all couples of points such that the segment between them does not contain the origin.

It is readily seen that whenever a smooth function $\psi:(0, a) \longrightarrow R$ is given such that the integral

$$
\int_{0}^{\rho} \frac{1}{2 \tau}\left(\tau \psi^{\prime}(\tau)-\psi(\tau)\right)^{2} \mathrm{~d} \tau
$$

is convergent at 0 , a smooth function $\varphi_{\psi}:[0, a) \longrightarrow R$ can be found such that the respective operator $S$ with radial velocity $\varphi_{\psi}$ and tangent velocity $\psi$ is a minimal monotone radially symmetric operator. Indeed,

$$
\varphi_{\psi}(\rho)=\sqrt{\int_{0}^{\rho} \frac{1}{2 \tau}\left(\tau \psi^{\prime}(\tau)-\psi(\tau)\right)^{2} \mathrm{~d} \tau}
$$

satisfies all requirements.

Proposition 2. If for a radially symmetric monotone operator the second inequality in (11) is strict on some compact interval $[c, d], c \geq 0$, then the operator is not acyclic with respect to $D=\left\{x \in R^{2}:\|x\| \leq d\right\}$.

Proof. Let

$$
S(x)=\varphi(\rho) \cdot \frac{x}{\rho}+\psi(\rho) \cdot \frac{\operatorname{rot}(x)}{\rho} .
$$


Then the second inequality in (1) (the operator $S$ is monotone) and the definition of $\varphi_{\psi}$ imply

$$
\begin{aligned}
\left(\varphi^{2}(\rho)\right)^{\prime} & =2 \varphi(\rho) \varphi^{\prime}(\rho) \geq \frac{1}{2 \rho}\left(\rho \psi^{\prime}(\rho)-\psi(\rho)\right)^{2} \\
& =\left(\int_{0}^{\rho} \frac{1}{2 \tau}\left(\tau \psi^{\prime}(\tau)-\psi(\tau)\right)^{2} \mathrm{~d} \tau\right)^{\prime}=\left(\varphi_{\psi}^{2}(\rho)\right)^{\prime} .
\end{aligned}
$$

As $\varphi(0)=0=\varphi_{\psi}(0)$ and both $\varphi$ and $\varphi_{\psi}$ take only nonnegative values (monotonicity with respect to the origin), we have $\varphi(\rho)-\varphi_{\psi}(\rho) \geq 0$ everywhere. The assumption of the proposition yields that the inequality in the chain of equalities and inequalities above is strict on $[c, d]$ and, hence, $\varphi(\rho)-\varphi_{\psi}(\rho)>0$ for $\rho \in(c, d]$; in particular, $\varphi(\rho)>0$ on $(c, d]$. We now define $\eta:[0, d] \longrightarrow[0,+\infty)$ by

$$
\eta(\rho)= \begin{cases}\varphi(\rho) & \text { for } \rho \in[0, c], \\ \varphi(c)+q(\varphi(\rho)-\varphi(c)) & \text { for } \rho \in[c, d],\end{cases}
$$

where the constant $q \in(0,1)$ can be chosen in such a way that the operator

$$
R(x)=\eta(\rho) \cdot \frac{x}{\rho}+\psi(\rho) \cdot \frac{\operatorname{rot}(x)}{\rho}
$$

is monotone. Indeed, $\eta$ is clearly continuous. The monotonicity of $S$ implies that $\varphi$ is increasing (because of the first inequality in (1)). Therefore $\eta$ is increasing as well. As $\eta$ coincides with $\varphi$ on $[0, c]$, the inequalities (11) are satisfied on this interval. When $\rho \in[c, d]$ we have $\eta^{\prime}(\rho)=q \varphi^{\prime}(\rho)$, and therefore

$$
\eta(\rho) \eta^{\prime}(\rho)=[\varphi(c)+q(\varphi(\rho)-\varphi(c))] q \varphi^{\prime}(\rho)=q^{2} \varphi(\rho) \varphi^{\prime}(\rho)+q(1-q) \varphi^{\prime}(\rho) \varphi(c) .
$$

As $\varphi(\rho) \varphi^{\prime}(\rho)-\varphi_{\psi}(\rho) \varphi_{\psi}^{\prime}(\rho) \geq \varepsilon_{0}>0$ on $[c, d]$, we can choose $q \in(0,1)$ as close to one that $\eta(\rho) \eta^{\prime}(\rho) \geq \varphi_{\psi}(\rho) \varphi_{\psi}^{\prime}(\rho)$ for every $\rho \in[c, d]$. Now we have $S=F+R$, where

$$
F(x)=(\varphi(\rho)-\eta(\rho)) \frac{x}{\rho} .
$$

The operator $F$ is not constant (since $\varphi$ is strictly positive on $(c, d]$ ), and $F=\partial f$ where $f(x)=\int_{0}^{\|x\|}(\varphi(\rho)-\eta(\rho)) \mathrm{d} \rho$. The function $f$ is convex because $\varphi^{\prime}(\rho) \geq$ $\eta^{\prime}(\rho)$, and hence $\varphi-\eta$ is increasing on $[0, d]$. Therefore the operator $S$ is not acyclic on $D$.

Thus, choosing $\psi(\rho)=-\frac{1}{\ln \rho}$ we obtain the following example:

$$
\mid \begin{aligned}
& \psi(\rho)=-\frac{1}{\ln \rho}, \\
& \varphi(\rho)=\sqrt{-\frac{1}{6(\ln \rho)^{3}}-\frac{1}{2(\ln \rho)^{2}}-\frac{1}{2 \ln \rho}} .
\end{aligned}
$$

This is a new explicit example of a nonlinear acyclic mapping because of the following:

Theorem 2. Let $S:$ dom $S \subset R^{2} \rightarrow R^{2}$ be a minimal monotone radially symmetric operator. Then it is an acyclic mapping.

The proof is contained in the next section. The example presented by Borwein and Wiersma in [3] satisfies all conditions in Definition 4 except for smoothness everywhere. Nevertheless the proof of Theorem 2 works for it. Note that the proof 
yields that if $S$ is a radially symmetric monotone operator and $4 \rho_{0} \varphi^{\prime}\left(\rho_{0}\right) \varphi\left(\rho_{0}\right)=$ $\left(\rho_{0} \psi^{\prime}\left(\rho_{0}\right)-\psi\left(\rho_{0}\right)\right)^{2}$ for some $\rho_{0}>0$ with $\varphi^{\prime}\left(\rho_{0}\right) \neq 0$, then $S$ is acyclic with respect to the set $D=\left\{x \in R^{2}:\|x\| \leq \rho_{0}\right\}$. Compare this with the statement of Proposition 2 to acknowledge the fact that the class of acyclic radially symmetric operators on the plane is strictly larger than the class of minimal radially symmetric operators.

\section{Proof of Theorem 2}

Let $S$ be a minimal monotone radially symmetric operator. Let $S=F+R$ where $R$ is monotone and $F=\partial f$ for some convex function $f$. Moreover, without loss of generality, let $F(0,0)=(0,0)$. We have to prove that $F \equiv(0,0)$.

The single-valuedness of $S$ implies that $F$ and $R$ are single-valued as well and, therefore, continuous, because they are monotone. It is straightforward that

$$
\lim _{t \rightarrow 0} \frac{\langle S(x+t l)-S(x), l\rangle}{t}=\langle\nabla S(x) . l, l\rangle
$$

for any $l \in R^{2}$ and any $x$ in the domain of $S$. We can think about this quantity as the directional derivative of $S$ in direction $l$. The minimality of $S$ implies that the matrix $\nabla S(x)$ is positive-semidefinite, but not positive-definite. Therefore for every $x$ not coinciding with the origin there exists a direction $l(x) \in R^{2},\|l(x)\|=1$ (and these directions are radially symmetric; that is, $l(x)$ depends only on $\|x\|$ ) such that the directional derivative of $S$ in direction $l(x)$ is zero:

$$
\lim _{t \rightarrow 0} \frac{\langle S(x+t l(x))-S(x), l(x)\rangle}{t}=0 .
$$

On the other hand, we have

$$
\begin{aligned}
\frac{\langle S(x+t l(x))-S(x), l(x)\rangle}{t}= & \frac{\langle F(x+t l(x))-F(x), l(x)\rangle}{t} \\
& +\frac{\langle R(x+t l(x))-R(x), l(x)\rangle}{t},
\end{aligned}
$$

and for all these operators ( $\tilde{S}$ is $S, F$ or $R$ ) monotonicity implies

$$
\frac{\langle\tilde{S}(x+t l(x))-\tilde{S}(x), l(x)\rangle}{t}=\frac{\langle\tilde{S}(x+t l(x))-\tilde{S}(x),(x+t l(x))-x\rangle}{t^{2}} \geq 0 .
$$

Hence $F$ and $R$ are directionally differentiable at $x$ in direction $l(x)$ with derivative zero, and the convergence rate is dominated by $S$. Indeed,

$$
0 \leq \frac{\langle F(x+t l(x))-F(x), l(x)\rangle}{t} \leq \frac{\langle S(x+t l(x))-S(x), l(x)\rangle}{t} \underset{t \rightarrow 0}{\longrightarrow} 0 .
$$

Therefore the convergence stated above is uniform with respect to $\theta \in[0,2 \pi]$ for any fixed $\rho$ because $S$ is radially symmetric (here $(\rho, \theta)$ are the polar coordinates of $x$ ).

Let $F$ have the appearance

$$
F(x)=\varphi_{F}(\rho, \theta) \frac{x}{\rho}+\psi_{F}(\rho, \theta) \frac{\operatorname{rot}(x)}{\rho} .
$$

Let us denote the polar coordinates of $x+t l(x)$ by $\left(\rho_{t}, \theta_{t}\right)$, and let $\beta$ be the angle between the vectors $x$ and $l(x)$; that is,

$$
\cos \beta=\left\langle l(x), \frac{x}{\rho}\right\rangle \quad \text { and } \quad \sin \beta=\left\langle l(x), \frac{\operatorname{rot}(x)}{\rho}\right\rangle .
$$


Note that $\rho_{t}, \theta_{t}-\theta$ and $\beta$ do not depend on the polar angle $\theta$ because $S$ (and therefore $l(x)$ ) is radially symmetric. We omit the argument $\rho$ of $\beta$ for the sake of shortness of the notation. Then we have

$$
\begin{aligned}
& 0=\lim _{t \rightarrow 0} \frac{\langle F(x+t l)-F(x), l(x)\rangle}{t} \\
& =\lim _{t \rightarrow 0} \frac{1}{t}\left\langle\varphi_{F}\left(\rho_{t}, \theta_{t}\right) \frac{x+t l(x)}{\rho_{t}}+\psi_{F}\left(\rho_{t}, \theta_{t}\right) \frac{\operatorname{rot}(x+t l(x))}{\rho_{t}}-\varphi_{F}(\rho, \theta) \frac{x}{\rho}\right. \\
& \left.-\psi_{F}(\rho, \theta) \frac{\operatorname{rot}(x)}{\rho}, l(x)\right\rangle \\
& =\lim _{t \rightarrow 0} \frac{1}{t}\left(\varphi_{F}\left(\rho_{t}, \theta_{t}\right) \frac{\rho}{\rho_{t}} \cos \beta+\varphi_{F}\left(\rho_{t}, \theta_{t}\right) \frac{t}{\rho_{t}}+\psi_{F}\left(\rho_{t}, \theta_{t}\right) \frac{\rho}{\rho_{t}} \sin \beta\right. \\
& \left.-\varphi_{F}(\rho, \theta) \cos \beta-\psi_{F}(\rho, \theta) \sin \beta\right) \\
& =\lim _{t \rightarrow 0}\left(\frac{1}{\rho_{t}} \varphi_{F}\left(\rho_{t}, \theta_{t}\right)+\cos \beta \cdot \frac{\rho}{\rho_{t}} \frac{\varphi_{F}\left(\rho_{t}, \theta_{t}\right)-\varphi_{F}(\rho, \theta)}{t}+\cos \beta \cdot\left(\frac{\rho}{t \rho_{t}}-\frac{1}{t}\right) \cdot \varphi_{F}(\rho, \theta)\right. \\
& \left.+\sin \beta \cdot \frac{\rho}{\rho_{t}} \cdot \frac{\psi_{F}\left(\rho_{t}, \theta_{t}\right)-\psi_{F}(\rho, \theta)}{t}+\sin \beta \cdot\left(\frac{\rho}{t \rho_{t}}-\frac{1}{t}\right) \cdot \psi_{F}(\rho, \theta)\right) \\
& =\lim _{t \rightarrow 0}\left(\frac{1}{\rho_{t}} \varphi_{F}\left(\rho_{t}, \theta_{t}\right)+\cos \beta \cdot \frac{\rho}{\rho_{t}} \cdot \frac{\rho_{t}-\rho}{t} \cdot \frac{\varphi_{F}\left(\rho_{t}, \theta_{t}\right)-\varphi_{F}(\rho, \theta)}{\rho_{t}-\rho}\right. \\
& -\cos \beta \cdot \frac{\rho_{t}-\rho}{t} \cdot \frac{1}{\rho_{t}} \cdot \varphi_{F}(\rho, \theta)+\sin \beta \cdot \frac{\rho}{\rho_{t}} \cdot \frac{\rho_{t}-\rho}{t} \cdot \frac{\psi_{F}\left(\rho_{t}, \theta_{t}\right)-\psi_{F}(\rho, \theta)}{\rho_{t}-\rho} \\
& \left.-\sin \beta \cdot \frac{\rho_{t}-\rho}{t} \cdot \frac{1}{\rho_{t}} \cdot \psi_{F}(\rho, \theta)\right) \\
& =\lim _{t \rightarrow 0}\left(\frac{1}{\rho_{t}} \varphi_{F}\left(\rho_{t}, \theta_{t}\right)+\cos \beta \cdot \frac{\rho}{\rho_{t}} \cdot \frac{\rho_{t}-\rho}{t} \cdot \frac{\varphi_{F}\left(\rho_{t}, \theta_{t}\right)-\varphi_{F}\left(\rho, \theta_{t}\right)}{\rho_{t}-\rho}\right. \\
& +\cos \beta \cdot \frac{\rho}{\rho_{t}} \cdot \frac{\rho_{t}-\rho}{t} \cdot \frac{\varphi_{F}\left(\rho, \theta_{t}\right)-\varphi_{F}(\rho, \theta)}{\rho_{t}-\rho}-\cos \beta \cdot \frac{\rho_{t}-\rho}{t} \cdot \frac{1}{\rho_{t}} \cdot \varphi_{F}(\rho, \theta) \\
& \left.+\sin \beta \cdot \frac{\rho}{\rho_{t}} \cdot \frac{\rho_{t}-\rho}{t} \cdot \frac{\psi_{F}\left(\rho_{t}, \theta_{t}\right)-\psi_{F}(\rho, \theta)}{\rho_{t}-\rho}-\sin \beta \cdot \frac{\rho_{t}-\rho}{t} \cdot \frac{1}{\rho_{t}} \cdot \psi_{F}(\rho, \theta)\right) .
\end{aligned}
$$

The uniform convergence with respect to $\theta$ now implies

$$
\begin{aligned}
& 0=\int_{0}^{2 \pi}\left(\lim _{t \rightarrow 0} \frac{\langle F(x+t l(x))-F(x), l(x)\rangle}{t}\right) \mathrm{d} \theta=\lim _{t \rightarrow 0}\left(\int_{0}^{2 \pi} \frac{\langle F(x+t l(x))-F(x), l(x)\rangle}{t} \mathrm{~d} \theta\right) \\
&=\lim _{t \rightarrow 0}\left(\frac{1}{\rho_{t}} \int_{0}^{2 \pi} \varphi_{F}\left(\rho_{t}, \theta_{t}\right) \mathrm{d} \theta\right.+\cos \beta \cdot \frac{\rho}{\rho_{t}} \cdot \frac{\rho_{t}-\rho}{t} \cdot \int_{0}^{2 \pi} \frac{\varphi_{F}\left(\rho_{t}, \theta_{t}\right)-\varphi_{F}\left(\rho, \theta_{t}\right)}{\rho_{t}-\rho} \mathrm{d} \theta \\
&+\cos \beta \cdot \frac{\rho}{\rho_{t}} \cdot \frac{\rho_{t}-\rho}{t} \cdot \int_{0}^{2 \pi} \frac{\varphi_{F}\left(\rho, \theta_{t}\right)-\varphi_{F}(\rho, \theta)}{\rho_{t}-\rho} \mathrm{d} \theta \\
&-\cos \beta \cdot \frac{\rho_{t}-\rho}{t} \cdot \frac{1}{\rho_{t}} \cdot \int_{0}^{2 \pi} \varphi_{F}(\rho, \theta) \mathrm{d} \theta \\
&+\sin \beta \cdot \frac{\rho}{\rho_{t}} \cdot \frac{\rho_{t}-\rho}{t} \cdot \int_{0}^{2 \pi} \frac{\psi_{F}\left(\rho_{t}, \theta_{t}\right)-\psi_{F}(\rho, \theta)}{\rho_{t}-\rho} \mathrm{d} \theta \\
&\left.-\sin \beta \cdot \frac{\rho_{t}-\rho}{t} \cdot \frac{1}{\rho_{t}} \cdot \int_{0}^{2 \pi} \psi_{F}(\rho, \theta) \mathrm{d} \theta\right) .
\end{aligned}
$$


We now use the fact that $F=\partial f$. Therefore the line integral of $F$ on a smooth closed curve (a circle centered at the origin in our case) is zero. Thus (because $\operatorname{rot}(x(\rho, \theta))$ is tangent to our circle) we have

$$
\begin{aligned}
0 & =\oint_{C_{\rho}} F_{1} \mathrm{~d} x_{1}+F_{2} \mathrm{~d} x_{2}=\int_{0}^{2 \pi}\langle F(x(\rho, \theta)), \operatorname{rot}(x(\rho, \theta))\rangle \mathrm{d} \theta \\
& =\rho \int_{0}^{2 \pi}\left\langle\varphi_{F}(\rho, \theta) \frac{x(\rho, \theta)}{\rho}+\psi_{F}(\rho, \theta) \frac{\operatorname{rot}(x(\rho, \theta))}{\rho}, \frac{\operatorname{rot}(x(\rho, \theta))}{\rho}\right\rangle \mathrm{d} \theta \\
& =\rho \int_{0}^{2 \pi} \psi_{F}(\rho, \theta) \mathrm{d} \theta .
\end{aligned}
$$

Since $\theta_{t}=\theta+$ const, we obtain

$$
\begin{aligned}
\int_{0}^{2 \pi} \psi_{F}\left(\rho_{t}, \theta_{t}\right) \mathrm{d} \theta & =\int_{0}^{2 \pi} \psi_{F}\left(\rho_{t}, \theta_{t}\right) \mathrm{d} \theta_{t} \\
& =\frac{1}{\rho_{t}} \int_{0}^{2 \pi}\left\langle F\left(x\left(\rho_{t}, \theta_{t}\right)\right), \operatorname{rot}\left(x\left(\rho_{t}, \theta_{t}\right)\right)\right\rangle \mathrm{d} \theta_{t}=0 .
\end{aligned}
$$

Therefore

$$
\begin{aligned}
\int_{0}^{2 \pi} & \frac{\psi_{F}\left(\rho_{t}, \theta_{t}\right)-\psi_{F}(\rho, \theta)}{\rho_{t}-\rho} \mathrm{d} \theta \\
& =\frac{1}{\rho_{t}-\rho}\left(\int_{0}^{2 \pi} \psi_{F}\left(\rho_{t}, \theta_{t}\right) \mathrm{d} \theta-\int_{0}^{2 \pi} \psi_{F}(\rho, \theta) \mathrm{d} \theta\right)=0 .
\end{aligned}
$$

Similarly we have

$$
\int_{0}^{2 \pi} \varphi_{F}\left(\rho, \theta_{t}\right) \mathrm{d} \theta=\int_{0}^{2 \pi} \varphi_{F}\left(\rho, \theta_{t}\right) \mathrm{d} \theta_{t}=\int_{0}^{2 \pi} \varphi_{F}(\rho, \theta) \mathrm{d} \theta
$$

and, hence,

$$
\begin{aligned}
\int_{0}^{2 \pi} & \frac{\varphi_{F}\left(\rho, \theta_{t}\right)-\varphi_{F}(\rho, \theta)}{\rho_{t}-\rho} \mathrm{d} \theta \\
\quad= & \frac{1}{\rho_{t}-\rho}\left(\int_{0}^{2 \pi} \varphi_{F}\left(\rho, \theta_{t}\right) \mathrm{d} \theta-\int_{0}^{2 \pi} \varphi_{F}(\rho, \theta) \mathrm{d} \theta\right)=0 .
\end{aligned}
$$

Thus, using (3), (4) and (5), equation (2) becomes

$$
\begin{aligned}
0=\lim _{t \rightarrow 0}\left(\frac{1}{\rho_{t}} \int_{0}^{2 \pi} \varphi_{F}\left(\rho_{t}, \theta_{t}\right) \mathrm{d} \theta+\cos \beta \cdot \frac{\rho}{\rho_{t}} \cdot \frac{\rho_{t}-\rho}{t} \cdot \int_{0}^{2 \pi} \frac{\varphi_{F}\left(\rho_{t}, \theta_{t}\right)-\varphi_{F}\left(\rho, \theta_{t}\right)}{\rho_{t}-\rho} \mathrm{d} \theta\right. \\
\left.-\cos \beta \cdot \frac{\rho_{t}-\rho}{t} \cdot \frac{1}{\rho_{t}} \cdot \int_{0}^{2 \pi} \varphi_{F}(\rho, \theta) \mathrm{d} \theta\right) .
\end{aligned}
$$

To pass to the limit in the above equality, we calculate

$$
\lim _{t \rightarrow 0} \frac{\rho_{t}-\rho}{t}=\lim _{t \rightarrow 0} \frac{\|x+t l(x)\|-\|x\|}{t}=\lim _{t \rightarrow 0} \frac{\sqrt{\rho^{2}+t^{2}+2 \rho t \cos \beta}-\rho}{t}=\cos \beta .
$$

On the other hand, with $\varphi_{F}$ being continuous on $(\rho-\varepsilon, \rho+\varepsilon) \times[0,2 \pi]$, we obtain

$$
\lim _{t \rightarrow 0}\left(\int_{0}^{2 \pi} \varphi_{F}\left(\rho_{t}, \theta_{t}\right) \mathrm{d} \theta_{t}\right)=\int_{0}^{2 \pi} \varphi_{F}(\rho, \theta) \mathrm{d} \theta .
$$


Thus (6) becomes (the argument $\rho$ of $\beta$ is explicitly written)

$$
0=\frac{\sin ^{2} \beta(\rho)}{\rho} \int_{0}^{2 \pi} \varphi_{F}(\rho, \theta) \mathrm{d} \theta+\cos ^{2} \beta(\rho) \cdot \lim _{t \rightarrow 0} \int_{0}^{2 \pi} \frac{\varphi_{F}\left(\rho_{t}, \theta_{t}\right)-\varphi_{F}\left(\rho, \theta_{t}\right)}{\rho_{t}-\rho} \mathrm{d} \theta .
$$

To finish the proof, we are going to use monotonicity of $F$ in radial directions:

$$
0 \leq\langle F(x)-F(0,0), x-(0,0)\rangle=\rho \cdot \varphi_{F}(\rho, \theta) .
$$

Let $\bar{x}$ be the vector with polar coordinates $\left(\rho, \theta_{t}\right)$ and $\bar{x}_{t}$ be the vector with polar coordinates $\left(\rho_{t}, \theta_{t}\right)$. Then

$$
0 \leq\left\langle F\left(\bar{x}_{t}\right)-F(\bar{x}), \bar{x}_{t}-\bar{x}\right\rangle=\left(\rho_{t}-\rho\right)^{2} \cdot \frac{\varphi_{F}\left(\rho_{t}, \theta_{t}\right)-\varphi_{F}\left(\rho, \theta_{t}\right)}{\rho_{t}-\rho} .
$$

Therefore

$$
\int_{0}^{2 \pi} \varphi_{F}(\rho, \theta) \mathrm{d} \theta \geq 0 \quad \text { and } \quad \lim _{t \rightarrow 0} \int_{0}^{2 \pi} \frac{\varphi_{F}\left(\rho_{t}, \theta_{t}\right)-\varphi_{F}\left(\rho, \theta_{t}\right)}{\rho_{t}-\rho} \mathrm{d} \theta \geq 0 .
$$

If $\sin \beta(\rho) \neq 0$, then the above inequalities and (77) imply

$$
\int_{0}^{2 \pi} \varphi_{F}(\rho, \theta) \mathrm{d} \theta=0
$$

and since $\varphi_{F}$ is continuous and nonnegative, this implies that $\varphi_{F}(\rho, \theta)=0$ for every $\theta \in[0,2 \pi]$. Note that $\beta$ depends only on $\rho$ and that the dependence in question is continuous since $S$ is smooth. If $\sin \beta(\rho)=0$ on an interval $\Delta$ with nonempty interior, this means that $l(x)$ is $x / \rho$ or $-x / \rho$. Since $F$ is directionally differentiable at $x(\rho, \theta)$ in direction $l(x)$ with derivative zero, the directional derivative of $F$ along $x / \rho$ exists and equals zero. This directional derivative coincides with the partial derivative $\left(\varphi_{F}\right)_{\rho}^{\prime}(\rho, \theta)$. Thus for $\rho \in \Delta$ we have $\left(\varphi_{F}\right)_{\rho}^{\prime}(\rho, \theta) \equiv 0$, and hence $\varphi_{F}(\rho, \theta)$ is constant with respect to $\rho$ on $\Delta$. The last two assertions, together with $F(0,0)=(0,0)$, prove that $\varphi_{F} \equiv 0$ on the domain of $S$.

Let $x=(\rho, \theta)$ and $\tilde{x}=(\rho, \tilde{\theta})$ have equal norms. Then monotonicity of $F$ implies

$$
\begin{aligned}
0 \leq & \langle F(\tilde{x})-F(x), \tilde{x}-x\rangle=\left\langle\psi_{F}(\rho, \tilde{\theta}) \frac{\operatorname{rot}(\tilde{x})}{\rho}-\psi_{F}(\rho, \theta) \frac{\operatorname{rot}(x)}{\rho}, \tilde{x}-x\right\rangle \\
& =\frac{\langle\operatorname{rot}(x), \tilde{x}\rangle}{\rho}\left(\psi_{F}(\rho, \tilde{\theta})-\psi_{F}(\rho, \theta)\right)=\frac{1}{\rho} \sin (\tilde{\theta}-\theta) \cdot\left(\psi_{F}(\rho, \tilde{\theta})-\psi_{F}(\rho, \theta)\right) .
\end{aligned}
$$

The above inequality shows that $\psi_{F}(\rho, \tilde{\theta})-\psi_{F}(\rho, \theta)$ and $\tilde{\theta}-\theta$ have the same sign whenever $|\tilde{\theta}-\theta|<\pi / 2$. Thus $\psi_{F}$ is increasing on the circle with radius $\rho$ centered at the origin, and therefore it is constant on this circle. Again using the fact that the line integral of $F$ on a circle centered at the origin is zero because of $F=\partial f$ (see (3)), we have $\int_{0}^{2 \pi} \psi_{F}(\rho, \theta) \mathrm{d} \theta=0$ and therefore $\psi_{F} \equiv 0$. Together with $\varphi_{F} \equiv 0$ this proves our theorem.

Remark. Note that most of the considerations above hold for a fixed $\rho_{0}>0$. Thus if $4 \rho_{0} \varphi^{\prime}\left(\rho_{0}\right) \varphi\left(\rho_{0}\right)=\left(\rho_{0} \psi^{\prime}\left(\rho_{0}\right)-\psi\left(\rho_{0}\right)\right)^{2}$ and $\varphi^{\prime}\left(\rho_{0}\right) \neq 0$, we have $\varphi_{F}\left(\rho_{0}, \theta\right)=0$ for every $\theta \in[0,2 \pi]$. But $\varphi_{F}(\cdot, \theta)$ is increasing with its value at the origin being zero. Therefore $\varphi_{F}(\rho, \theta)=0$ for every $\theta \in[0,2 \pi]$ and for every $\rho \leq \rho_{0}$, thus justifying the statement at the end of the second section. 


\section{REFERENCES}

[1] E. Asplund, A monotone convergence theorem for sequences of nonlinear mappings, Proc. Sympos. Pure Math., 18, Amer. Math. Soc., Providence, RI (1970), pp. 1-9. MR0275240 $(43: 997)$

[2] J.M. Borwein, Maximal monotonicity via convex analysis, J. Convex Anal., 13(2006), pp. 561-586. MR.2291552 (2008d:47096)

[3] J.M. Borwein, H. Wiersma, Asplund decomposition of monotone operators, SIAM J. Optim., 18(2007), pp. 946-960. MR2345978 (2009i:47107)

[4] R.T. Rockafellar, Characterization of the subdifferentials of convex functions, Pacific J. Math., 17(1966), pp. 497-510. MR0193549 (33:1769)

[5] R.T. Rockafellar, R.J.-B. Wets, Variational Analysis, Springer, 2004. MR1491362 (98m:49001)

Department of Mathematics and Informatics, University of Sofia, James Bourchier Boulevard 5, 1126 Sofia, Bulgaria - and - Institute of Mathematics and Informatics,

BAS, G. Bonchev str. 8, 1113 Sofia, Bulgaria

E-mail address: boilbulgarian@gmail.com

Department of Mathematics and Informatics, University of Sofia, James Bourchier Boulevard 5, 1126 Sofia, Bulgaria - And - Institute of Mathematics and Informatics,

BAS, G. Bonchev str. 8, 1113 Sofia, Bulgaria

E-mail address: ribarska@fmi.uni-sofia.bg 